Fayetteville State University

DigitalCommons@Fayetteville State University

Natural Sciences Faculty Working Papers

College of Arts and Sciences

$1-5-2007$

\title{
Identification of Material Parameters for Continuum Modeling of Phase Transformations In Multicomponent Systems
}

Alexander Umantsev

Fayetteville State University, aumantsev@uncfsu.edu

\section{Recommended Citation}

Umantsev, Alexander, "Identification of Material Parameters for Continuum Modeling of Phase Transformations In Multicomponent Systems" (2007). Natural Sciences Faculty Working Papers. Paper 13.

http://digitalcommons.uncfsu.edu/natsci_wp/13

This Article is brought to you for free and open access by the College of Arts and Sciences at DigitalCommons@Fayetteville State University. It has been accepted for inclusion in Natural Sciences Faculty Working Papers by an authorized administrator of DigitalCommons@Fayetteville State University. For more information, please contact mlawson@uncfsu.edu. 


\title{
Identification of material parameters for continuum modeling of phase transformations in multicomponent systems
}

\author{
Alex Umantsev \\ Department of Natural Sciences, Fayetteville State University, 1200 Murchison Road, Fayetteville, North Carolina 28301, USA
}

(Received 26 January 2006; published 5 January 2007)

\begin{abstract}
The continuum (field theoretic) method has become the method of choice for multiscale structure-formation modeling of very different phase transformations in the past decade. One of the challenges in application of the method to transformations in real materials is to obtain the mesoscopic parameters, which characterize the thermodynamic system of interest. Significant progress has been made in the case of pure systems; however, one would like to know what changes need to be made in the case of binary or multicomponent systems. We consider an exactly solvable case of the linear multicomponent system undergoing a phase transformation and derive equations that relate parameters of the continuum method, like barrier height, gradient energy, and relaxation coefficients, to the measurable quantities, like interface energy, interfacial thickness, and kinetic coefficient. We find that the contribution of chemical interactions in the system can be expressed as the renormalization of the barrier-height parameter of the continuum method and replacement of the latent heat with the chemical modulus. Atomic-scale simulations data for a solid/liquid transition in a binary $\mathrm{Cu}-\mathrm{Ni}$ system were chosen for comparison with the theory and the fitting yields the estimates for the continuum-method parameters. Analysis of the temperature dependence of the interfacial energy allowed us to shed light on the magnitudes of the internal energy and entropy contributions into the solid/liquid interface.
\end{abstract}

DOI: 10.1103/PhysRevB.75.024202 PACS number(s): 64.10.+h, 64.70.Dv, 68.03.Cd, 68.08.-p

\section{INTRODUCTION}

In the past decade the continuum (field theoretic) method has become very popular in theoretical and computational studies of very different phase transformations in materials. The success of the method is due to its computational flexibility and ability to transcend the constraints of spatial/ temporal scales, imposed by strictly microscopic or macroscopic methods, hence becoming a truly multiscale one. Although phase transitions in materials are always associated with sharp changes of properties, according to the Landau theory, ${ }^{1}$ they may be characterized by one or more coarsegrained continuous variables, $\eta_{l}$, commonly called order parameters, which take on specified values in the bulk phases. If the transformation is accompanied by redistribution of species, there will be another set of continuous (field) variables, the partial densities of different components, $\rho_{1}, \rho_{2}, \rho_{3}, \ldots$, which also characterize the free energy of the material: $F=F\left(\eta_{l}, \rho_{i}\right)$. The presence of interfaces makes our system inhomogeneous, that is, there appear gradients of the independent variables, densities, and order parameters. This affects the free energy in two ways. First, it becomes a functional over the entire system: $F=\int \hat{f} d^{3} x$. Second, the density of the free energy, $\hat{f}$, becomes a function of the gradients of the thermodynamic variables as well as variables themselves, ${ }^{2}$

$\hat{f}=f_{\alpha}\left(T ; \rho_{i}\right)+\Delta f\left(T ; \rho_{i}, \eta\right)+\frac{1}{2} \nabla \rho_{i} \kappa_{i j}(T) \nabla \rho_{j}+\frac{1}{2} \kappa(T)(\nabla \eta)^{2}$.

We consider here the free energy density to be a function of a single-order parameter in the so-called square-gradient approximation; $f_{\alpha}$ is the free energy density of $\alpha$-phase, the Landau function $\Delta f$ reflects symmetries of $\alpha$ and $\beta$ phases and thermodynamic properties of the transition between them; $\left\{\kappa_{i j}\right\}$ is the symmetric matrix of the compositional gradient free energy coefficients and $\kappa$ is the order-parameter gradient free energy coefficient. The free energy of phases and the gradient energy coefficients may be functions of temperature and pressure. In this paper temperature will be considered uniform throughout the system but varying from one situation to another; the pressure, however, will be considered constant for all situations.

If the system is set up away from equilibrium, it will be evolving in the direction of the equilibrium state due to the presence of the thermodynamic driving forces, which are expressed as the variational derivatives of the free energy with respect to its variables. As the order parameters do not obey any conservation constrains, their evolution equations are of nonconservative type and are known as the time-dependent Ginzburg-Landau equation (TDGLE):

$$
\frac{d \eta}{d t}=-\gamma\left(\frac{\delta F}{\delta \eta}\right) T
$$

Here and below $d / d t$ means the substantial derivative, and $\gamma$ is the relaxation coefficient, which sets the time scales of the process.

As we pointed out above, the diffusional transformations are characterized by another set of variables, densities $\rho_{i}$, which may also undergo changes together with the order parameters. The fundamental difference between the former and the latter is in the conservation constraints that the densities must obey. This difference is translated into the different type of evolution equations, that is, diffusion equations where the mass fluxes are proportional to the chemical potential gradients: $\mathbf{J}_{i} \propto \nabla\left(\delta F / \delta \rho_{i}\right)$. Then the diffusion equation takes the form 


$$
\frac{d \rho_{i}}{d t}=\nabla\left[M_{i j}\left(T ; \rho_{k}, \eta\right) \cdot \nabla \frac{\delta F}{\delta \rho_{j}}\right]
$$

Here $\left\{M_{i j}\right\}$ is the matrix of solute mobility coefficients. The system of coupling diffusion-type equations, Eqs. (2) and (3), describes dynamics of the system.

A continuum method to study equilibrium properties of interfaces in multicomponent systems was developed in Ref. 3 . For the free energy density, Eq. (1), the equilibrium in the heterogeneous system may be expressed by the following equations:

$$
\begin{gathered}
\kappa \nabla^{2} \eta=\partial_{\eta} f ; \\
\kappa_{i j} \nabla^{2} \rho_{j}=\partial_{\rho_{t}} f-\mu_{i} ; \\
f\left(T, \rho_{i}, \eta\right)-\mu_{i} \rho_{i}-\frac{1}{2} \frac{d \rho_{i}}{d x} \kappa_{i j} \frac{d \rho_{j}}{d x}-\frac{1}{2} \kappa\left(\frac{d \eta}{d x}\right)^{2}=\text { const. }
\end{gathered}
$$

In Eq. (4b) $\mu_{i}$ are the chemical potentials; Eq. (4c) applies only to one-dimensional (1D) systems, e.g., plane interfaces, and the constant in this equation relates to the hydrostatic pressure. Then, using a solution of Eq. (4c), the interfacial energy of a plane interface may be expressed as follows: ${ }^{3,4}$

$$
\sigma=\int_{\alpha}^{\beta}\left\{\frac{d \rho_{i}}{d x} \kappa_{i j} \frac{d \rho_{j}}{d x}+\kappa\left(\frac{d \eta}{d x}\right)^{2}\right\} d x .
$$

Here the integration covers not only the interfacial region but extends over the contiguous bulk phases $\alpha$ and $\beta$ also. The condition of stability of homogeneous phases requires the surface energy $\sigma$ to be positive. ${ }^{2}$ Application of the matrix theory to Eq. (5) yields that for the stability of phases all eigenvalues of the matrix $\left\{\kappa_{i j}\right\}$ and the order-parameter gradient free energy coefficient $\kappa$ must be non-negative. ${ }^{25}$

The central point of the theory in Ref. 3 is the Gibbs adsorption equation in the continuum representation. If temperature $T$ and chemical potentials $\mu_{l}$ are taken as independent variables, the Gibbs adsorption equation takes the following form:

$$
d \sigma=-\Gamma_{S}^{(1)} d T-\Gamma_{l}^{(1)} d \mu_{l} ; \quad l=2, \ldots, n .
$$

Here $\Gamma_{S}^{(1)}$ is the relative surface entropy and $\Gamma_{l}^{(1)}$ is the adsorption of component $l$ with respect to the component 1 .

The free energy, Eq. (1), and the dynamic equations, Eqs. (2) and (3), contain two kinds of material parameters: bulk, which characterize the free energy of equilibrium phases and kinetic processes therein, and interfacial, which characterize the transformation between the phases, e.g., the energy scale of the Landau function, the gradient free energy coefficients, $\kappa$ and $\kappa_{i j}$, and the relaxation coefficient $\gamma$. The former ones may be easily obtained from the thermodynamic and kinetic databases, e.g., Thermocalc/Dictra ${ }^{\circledR}$; it is significantly more difficult to obtain the latter ones. This presents one of the challenges of the continuum method. The interfacial material parameters may be either obtained from first-principles calculations, bottom-up approach, or extracted from experiments, real or numerical, top-to-bottom approach. For quan- titatively meaningful analysis of the microstructure one needs to know the interfacial parameters as accurately as possible, regardless of the fact that due to computational reasons, numerical simulations usually cannot be conducted with the realistic parameters. As known, in the case of a pure system the interfacial parameters may be determined from the data on the measurable quantities: equilibrium interfacial energy, $\sigma$, equilibrium interfacial thickness, $\Delta$, and the thermal kinetic coefficient, $k_{T}{ }^{2,4,5}$ One would like to know what measures should be taken in order to identify the interfacial parameters in binary or multicomponent systems. In this case, for instance, there must be a solution thermodynamics contribution into the interfacial energy; as far as the interfacial thickness is concerned the very definition of this quantity must be reconsidered.

A number of authors used the top-to-bottom approach to a binary $\mathrm{Cu}-\mathrm{Ni}$ system. ${ }^{6-10}$ Wheeler et $a .^{6}{ }^{6}$ and Ahmad et al. ${ }^{7}$ assumed both phases (solid and liquid) to be ideal solutions of their elements and all the measurable interfacial properties of $\mathrm{Cu}, \mathrm{Ni}$, and the alloys of different compositions to be equal. Kim et al. ${ }^{8}$ introduced a binary alloy model which treats the interfacial region as a mixture of solid and liquid phases with the equilibrium concentrations but variable fraction - a mushy-zone model of sorts. In this approach the solution thermodynamics contribution into the interfacial energy vanishes due to the assumed equilibrium between the phases. Granasy et al. ${ }^{9}$ took a different approach: they fixed the parameters of the pure $\mathrm{Cu}$ and $\mathrm{Ni}$ separately and linearly approximated in temperature the parameters for the alloy system. Although the solution thermodynamics contribution can be recovered in this heuristic approach in the form of temperature dependence of the interfacial energy, the problem is that this temperature dependence may not match the experimental data. Echebarria et al. ${ }^{10}$ introduced a dilute binary alloy model which allows an exact solution for the equilibrium state. The assumptions of the model, however, make it virtually a pure-material system and do not allow recovering any temperature dependence of the interfacial energy which usually appears in alloys.

It is the intention of this work to lay out a thermodynamically self-consistent approach to the problem of identification of material parameters for continuum modeling of phase transformations in multicomponent systems. The main idea of the approach is to formulate a reasonable thermodynamic model of a binary or multicomponent alloy and obtain exact expressions for the equilibrium thermodynamic functions, like interfacial energy and thickness. Unfortunately, a very popular ideal-solution model ${ }^{11}$ does not allow for an exact solution. That is why in Sec. II we analyze a linear multicomponent system, introduced in Ref. 3, and apply this model to a binary $\mathrm{Cu}-\mathrm{Ni}$ system in Sec. III. We do not expect real alloy systems to be linear; we expect, however, the linearity to the first approximation to the real solution thermodynamics. Then, the derived expressions for the interfacial parameters may serve as the first approximations to the real ones. This conjecture will be tested in Sec. III by comparing the ideal solution model to the linear one.

Dynamics of phase transformations merits special consideration. One can prove rigorously that when the continuum (diffuse-interface) approach is applicable, the rate of motion 
of a plane boundary is proportional to the driving forcedeviation of the thermodynamic conditions on the boundary from the equilibrium ones. ${ }^{5,12}$ In case of pure substances the driving force has been identified as the deviation of the interface temperature from the equilibrium one and the speed of the boundary is equal to: $V=k_{T}\left(T-T_{E}\right)$. The stationary plane-front solution can be matched to an experiment under similar conditions and the experimentally measurable kinetic coefficient $k_{T}$ can be related to the theoretical quantity, relaxation parameter $\gamma$ of Eq. (2), $5,7,13$

$$
\gamma=\frac{2 k_{T} T_{E}}{3 \Delta L} .
$$

Here $L$ is the latent heat of the transformation.

The matching of the theoretical and experimental results is more complicated in alloys: although it can be worked out through the thermal kinetic coefficient, we believe that another approach is sounder. According to this approach (see Ref. 14 and references therein), the driving force is the deviation of the concentration in one of the phases from the equilibrium value with the coefficient of proportionality being the solute kinetic coefficient $k_{C}$, which needs to be related to the relaxation coefficient $\gamma$. In Sec. IV a binary linear model will be applied to a dynamical situation and the expression for the relaxation coefficient will be derived. The conclusions will be drawn in Sec. V.

\section{LINEAR MULTICOMPONENT SYSTEM}

A simple model of a multicomponent system which still has all the properties essential for phase transformations in real materials was introduced in Ref. 3. The model was called "linear," with the conditions of linearity as follows:

$$
\frac{\partial^{3} \hat{f}}{\partial \rho_{i} \partial \rho_{j} \partial \rho_{k}}=0 ; \quad \frac{\partial^{2} \hat{f}}{\partial T \partial \rho_{i}}=0 .
$$

Similar to the ideal solution model,,${ }^{11}$ the linear model does not allow for the ternary density interactions; however, different from the ideal model, the linear one does not allow for the interactions of density and thermal modes, with the greatest discrepancy between the two models near the puresubstance boundaries.

For the linear multicomponent system the relative adsorptions, $\Gamma_{l}^{(1)}$, vanish and the relative surface entropy takes the form $^{3}$

$$
\Gamma_{s}^{(1)}=-\frac{1}{2} \int_{\alpha}^{\beta}\left\{\frac{d \rho_{i}}{d x} \frac{d \kappa_{i j}}{d T} \frac{d \rho_{j}}{d x}+\frac{d \kappa}{d T}\left(\frac{d \eta}{d x}\right)^{2}\right\} d x .
$$

In the present paper a special consideration is made for the linear system with vanishing compositional gradient free energy coefficients, $\kappa_{i j}=0$. The author is not aware of any material system where the estimates of separate compositional and ordering contributions into the surface energy were made. However, estimates of these contributions in different systems show that usually the ordering one (e.g., liquid/solid in Cu-Ni, $\sigma=0.278 \mathrm{~J} / \mathrm{m}^{2}$, Ref. 19) is an order of magnitude greater than the compositional one (e.g., spinodal decomposition in Ag-Al, $\sigma=0.015 \mathrm{~J} / \mathrm{m}^{2}$, Ref. 24). This fact may justify consideration of a linear system with vanishing compositional gradient free energy coefficients, $\kappa_{i j}=0$. Many experimental and numerical techniques allow plotting variation of the concentration and order parameter across the interface. As one can see from Eqs. (4), the chemical and ordering length scales will coincide if $\kappa_{i j}=0$. Thus, comparison of the length scales of the variations of the order parameter and concentration provides a test for the magnitude of $\kappa_{i j}$. Direct comparison of these coefficients will be also discussed in Sec. III.

Comparison of Eq. (5) with Eq. (9) yields that for the system with $\kappa_{i j}=0$ the Gibbs adsorption equation, Eq. (6), takes the form

$$
d \sigma=\frac{\sigma}{2 \kappa} \frac{d \kappa}{d T} d T .
$$

Equation (10) can be easily integrated to yield

$$
\sigma^{2}=\text { const } \cdot \kappa(T) \text {. }
$$

Thus, the linear multicomponent model with vanishing compositional gradient free energy coefficients turns out to be exactly solvable. In the framework of the linear model the relation between the interfacial energy and gradient energy coefficient is independent of the details of the free energy function, Eq. (1); the latter affects only the magnitude of the constant of proportionality in Eq. (11).

One can show that under the conditions of linearity, Eq. (8), the free energy density of $\alpha$-phase in Eq. (1) can be represented as follows:

$$
f_{\alpha}\left(T ; \rho_{i}\right)=f_{\alpha, 0}(T)+\frac{1}{2} \rho_{i} N_{i j} \rho_{j} .
$$

where $\left\{N_{i j}\right\}$ is a symmetric nonsingular matrix of temperature-independent binary-interaction coefficients. There is extensive literature on the type of Landau function which should be used for the description of phase transitions of specific kinds; ${ }^{15}$ however, only one definite constraint on this function exists: it should have as many minima as there are stable phases in the system. The function employed in the present paper is the following: ${ }^{16}$

$$
\begin{aligned}
& \Delta f\left(T ; \rho_{i}, \eta\right)=\frac{1}{2} a \omega^{2}(\eta)+b\left(T ; \rho_{i}\right) \nu(\eta) \\
& \omega=\eta(1-\eta) ; \quad \nu=\eta^{2}(3-2 \eta) \\
& a=\mathrm{const} ; \quad b=f_{\beta}\left(T ; \rho_{i}\right)-f_{\alpha}\left(T ; \rho_{i}\right) .
\end{aligned}
$$

For this function the condition of phase equilibrium, Eq. (4a), yields that the values of the order parameters in bulk phases, $\eta_{\alpha}=0$ and $\eta_{\beta}=1$, are independent of the phase composition or temperature. The bulk phases are separated by the free energy barrier, whose coordinate is $\eta^{*}=1 / 2+3 b / a$. Parameter $a$ characterizes the curvature of the "potential well" and the free energy barrier height (if $b=0$ ) while parameter $b$ characterizes the driving force of transformation if the system is driven away from the equilibrium. The composition and temperature dependence of the parameter $b$ may be re- 
vealed through the conditions of linearity, Eq. (8), imposed on the Landau function, Eq. (13):

$$
b=b_{0}(T)+b_{i} \rho_{i} ; \quad b_{i}=\text { const } .
$$

Application of the conditions of chemical and mechanical equilibrium of the bulk phases, Eqs. (4b) and (4c), to the free energy, Eqs. (1) and (12)-(14), yields the following relations:

$$
\begin{gathered}
N_{i j} \rho_{j \alpha}=\mu_{i} ; \quad N_{i j}\left[\rho_{j}\right]=-b_{i} ; \quad \rho_{j \alpha}, \rho_{j \beta} \geq 0 ; \\
b_{0}+b_{i} \rho_{i \beta}=-r ; \quad r \equiv \frac{1}{2}\left[\rho_{i}\right] N_{i j}\left[\rho_{j}\right] .
\end{gathered}
$$

The quantities in square brackets are defined as $[\varphi] \equiv \varphi(\beta)$ $-\varphi(\alpha)$ and may be called the interfacial jump quantities. The constraint of non-negative densities appears because of nonsingular behavior of the free energy at $\rho_{i}=0$ [see Eq. (12)]. Notice that in the linear approximation $\rho_{i \alpha}$ and $\rho_{i \beta}$ are functions of temperature but $\left[\rho_{i}\right]$ is independent of temperature. The quantity $r$ is also temperature independent; it characterizes the multicomponent phase diagram and may be called the chemical modulus..$^{17}$ The latent heat of the linear system has the following very simple expression:

$$
L \equiv\left\{T \frac{\partial}{\partial T}-1\right\}[\hat{f}]=r-b_{0}+T \frac{d b_{0}}{d T} .
$$

Application of the condition of heterogeneous chemical equilibrium, Eq. (4b), to the free energy, Eqs. (1) and (12)(15), yields the following relation:

$$
\rho_{i}=\rho_{i \alpha}+\left[\rho_{i}\right] \nu(\eta)
$$

Then the condition of heterogeneous mechanical equilibrium, Eq. (4c), takes the form

$$
\kappa\left(\frac{d \eta}{d x}\right)^{2}=a \omega^{2}+2 r \nu(1-\nu) .
$$

[For the details of the derivation of Eq. (17) see Appendix.]

To obtain the expression for $\sigma$ one can substitute Eq. (17) into Eq. (5) and integrate it straightforwardly. However, to elucidate the contribution of solution thermodynamics into the surface energy, it is advantageous to notice that in the domain $0 \leq \eta \leq 1$ the function $1 / \omega$ varies rapidly and diverges at $\eta=0 ; 1$, while the function $H \equiv \nu(1-\nu) / \omega^{2}=(3$ $-2 \eta)(1+2 \eta)$ varies slowly between the values of 3 and 4 . Hence, according to Laplace's theorem, ${ }^{18}$ for the purposes of integration of Eq. (17), function $H$ can be replaced by its value at the peak points, $H \approx H(0)=H(1)=3$. Then Eq. (17) may be replaced by its asymptotic equivalent

$$
\kappa\left(\frac{d \eta}{d x}\right)^{2}=(a+6 r) \omega^{2} .
$$

This expression is practically identical to the case of elemental substances; the only difference is that the barrier-height parameter $a$ is renormalized to $(a+6 r)$. Then, from Eq. (5) the interfacial energy can be found to have the following expression:

$$
\sigma=\frac{1}{6} \sqrt{\kappa(a+6 r)}
$$

This relation is one of the two main results of the present paper. It is consistent with the Gibbs adsorption equation, Eq. (11), where const $=(a+6 r) / 36$.

Another quantity important for calculation of the parameters of the continuum method is the interfacial thickness, $\Delta$, the proper definition of which should be based on the length scale of the order parameter variation. One can define the interfacial thickness as $\Delta \equiv|[\eta]| / \max |d \eta / d x|,{ }^{2,5,13}$ which in the case of a simple "tanh" solution corresponds to a "12-88" interval. Then, from Eq. (18) it follows that the interfacial thickness is

$$
\Delta=4 \sqrt{\frac{\kappa}{a+6 r}} .
$$

Expressions for $\sigma$ and $\Delta$ in Eqs. (19) and (20) can be resolved for $a$ and $\kappa$,

$$
\kappa=\frac{3}{2} \sigma \Delta ; \quad a=6\left(4 \frac{\sigma}{\Delta}-r\right) .
$$

As one can see from Eq. (21), compared to the case of elemental systems, the multicomponent interactions change the expression for the barrier-height parameter $a$ but do not change the expression for $\kappa$.

\section{Cu-Ni BINARY SYSTEM}

One of the best examples of a linear-solution system is a liquid-solid transition in a binary $\mathrm{Cu}-\mathrm{Ni}$ alloy, with the primary reason for the linearity being the similarity of the elements. In the case of a binary system it is more convenient to use the mole fraction of one component, $c=\rho_{\mathrm{Cu}} /\left(\rho_{\mathrm{Cu}}+\rho_{\mathrm{Ni}}\right)$, instead of the densities of both. As one can see from Eqs. (12), (14), and (15), in the linear-solution approximation the free energies of the liquid $(l)$ and solid $(s)$ phases are the parabolic functions of concentration $c$ :

$$
f_{l(s)}(T ; c)=f_{l(s), 0}(T)+f_{l(s), 1} c+\frac{1}{2} f_{l(s), 2} c^{2}, \quad 0 \leq c \leq 1,
$$

where the second-order coefficients are equal: $f_{l, 2}=f_{\mathrm{s}, 2} \equiv f_{2}$. Then application of Eq. (15) to the free energy, Eqs. (1) and (22), yield

$$
\begin{aligned}
& C_{l}=\frac{\left[f_{1}\right]}{2 f_{2}}-\frac{\left[f_{0}\right]}{\left[f_{1}\right]} ; \quad C_{s}=-\frac{\left[f_{1}\right]}{2 f_{2}}-\frac{\left[f_{0}\right]}{\left[f_{1}\right]} ; \\
& 0 \leq C_{l}, C_{s} \leq 1 ; \quad r \equiv \frac{1}{2} f_{2}[C]^{2}=\frac{\left[f_{1}\right]^{2}}{2 f_{2}}
\end{aligned}
$$

Notice that in the linear approximation the liquidus $C_{l}$ and solidus $C_{s}$ are functions of temperature, while the concentration range $[C]$ is temperature independent. The phase diagram of the linear system envelops the phase diagram of the ideal solution. ${ }^{11}$ In the phase space of heterogeneous states 


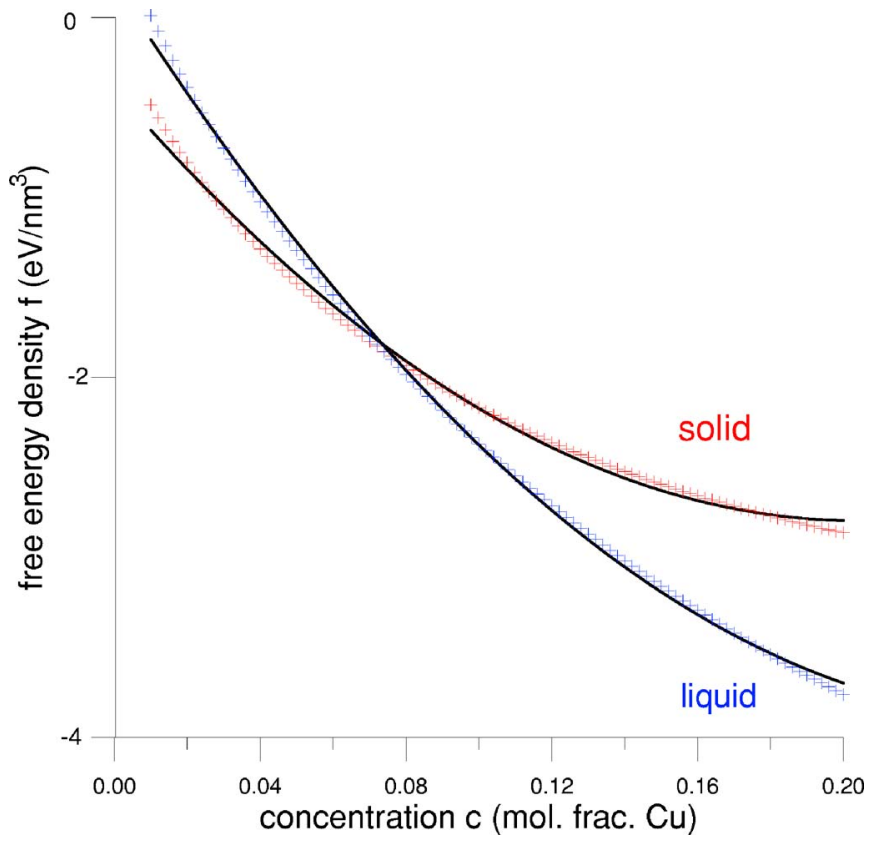

FIG. 1. (Color online) Fitting of the free energy densities of the solid and liquid phases of $\mathrm{Cu}-\mathrm{Ni}$ alloy at $T=1750 \mathrm{~K}$ into the second-order polynomial forms. Crosses are the numerical simulation data from Refs. 19 and 20, solid lines are the polynomial approximation.

the equilibrium concentrations, $C_{l}$ and $C_{s}$, are connected by the trajectory of the representative point, Eqs. (16) and (18). For a binary system projection of this trajectory on the $(\mathrm{c}, \eta)$ plane takes the form

$$
C=C_{l}+[C] \nu(\eta)
$$

The equilibrium trajectory crosses the barrier at the saddle point $\left(c^{*}, \eta\right)$ described by

$$
C^{*}=\frac{C_{s}+C_{l}}{2}+\frac{a}{3 f_{2}[C]}\left(\frac{1}{2}-\eta\right) .
$$

On the trajectory of the representative point [Eqs. (18) and (24)], the free energy density of the system [Eq. (1)] can be expressed as the function of the order parameter only,

$$
\hat{f}=f_{l}\left(T ; C_{l}\right)+\left(f_{l, 1}+f_{2} C_{l}\right)[C] \nu(\eta)+(a+6 r) \omega^{2}(\eta) .
$$

Asta et $a l .{ }^{19}$ have conducted atomic-scale simulations of the liquid-solid transition in $\mathrm{Cu}-\mathrm{Ni}$ systems and calculated the interfacial free energy at a temperature of $1750 \mathrm{~K}$, which is inside the two-phase range. For the purposes of the present analysis it would have been advantageous to have the simulation data at several different temperatures; unfortunately, so far the simulations have been finished only for $T=1750 \mathrm{~K}$. Below we will compare their numerical results with the theoretical model developed in the present paper in order to extract the parameters of the continuum method. The free energies of the phases were fitted into the polynomial forms, Eq. (22), in the range $0.01<c<0.2$ (see Fig. 1). In order to obtain the free energy densities, $f_{l(s)}$, the molar Gibbs free energies used for simulations of liquid/solid equilibrium in $\mathrm{Cu}-\mathrm{Ni}$ system $^{19,20}$ were multiplied by respective densities of the solid and liquid phases at the temperature of the simulations; ${ }^{19}$ the fitting results are shown in Table I. The difference between $f_{l, 2}$ and $f_{\mathrm{s}, 2}$ was less than $0.4 \%$, which supported the application of the linear-solution model to the $\mathrm{Cu}-\mathrm{Ni}$ system. The geometric average value, $f_{2}=\sqrt{ }\left(f_{l, 2} f_{\mathrm{s}, 2}\right)$, was used for all the calculations of the parameters. The interfacial thickness $\Delta$ was determined from the concentration profile provided in Ref. 19 instead of the order-parameter profile. Comparison with the latter is desirable but was not available. Figure 2 shows the phase diagram of the $\mathrm{Cu}-\mathrm{Ni}$ system for small concentrations of $\mathrm{Cu}$, as represented by the linear-solution model [color lines, Eq. (23)].

There are many different ways that one can use the parameters of the continuum method. One way is to apply them to real-material multiscale structure-formation modeling. Another way to use the parameters is to gain better understanding of the physics of the transformation process itself. Figure 3 is the $(c, \eta)$-plane of the phase space; the trajectory of the representative point of the equilibrium state, Eq. (24), is depicted in Fig. 3 together with the coordinates of the free

\begin{tabular}{|c|c|c|c|c|c|c|c|}
\hline \multicolumn{8}{|c|}{ Simulation } \\
\hline \multicolumn{3}{|c|}{ Solid phase } & \multicolumn{3}{|c|}{ Liquid phase } & \multicolumn{2}{|c|}{ Interface } \\
\hline$f_{0}$ & $f_{1}$ & $f_{2}$ & $f_{0}$ & $f_{1}$ & $f_{2}$ & $\sigma$ & $\Delta$ \\
\hline $\mathrm{eV} / \mathrm{nm}^{3}$ & $\mathrm{eV} / \mathrm{nm}^{3}$ m.f. & $\mathrm{eV} / \mathrm{nm}^{3} \mathrm{~m} . f .^{2}$ & $\mathrm{eV} / \mathrm{nm}^{3}$ & $\mathrm{eV} / \mathrm{nm}^{3}$ m.f. & $\mathrm{eV} / \mathrm{nm}^{3}{\mathrm{~m} . \mathrm{f}^{2}}^{2}$ & $\mathrm{eV} / \mathrm{nm}^{2}$ & $\mathrm{~nm}$ \\
\hline-0.4127 & -24.476 & 60.0093 & 0.18282 & -30.706 & 59.8262 & 1.792 & 0.75 \\
\hline \multicolumn{8}{|c|}{ Theory } \\
\hline & Solid & Liquid & \multicolumn{5}{|c|}{ Interface } \\
\hline & $C$ & $C$ & $r$ & $a$ & $\kappa$ & $\kappa_{e}$ & $\kappa_{s}$ \\
\hline & m.f. & m.f. & $\mathrm{eV} / \mathrm{nm}^{3}$ & $\mathrm{eV} / \mathrm{nm}^{3}$ & $\mathrm{eV} / \mathrm{nm}$ & $\mathrm{eV} / \mathrm{nm}$ & $\mathrm{eV} / \mathrm{nm} \mathrm{K}$ \\
\hline & 0.0436 & 0.1476 & 0.324 & 54.7 & 2.02 & -3.05 & -0.00290 \\
\hline
\end{tabular}
energy barrier, Eq. (25). Also in Fig. 3 is depicted the free

TABLE I. Fitting of the simulation data for the solid/liquid $\mathrm{Cu}-\mathrm{Ni}$ system at $T=1750 \mathrm{~K}$ into the theoretical model. 


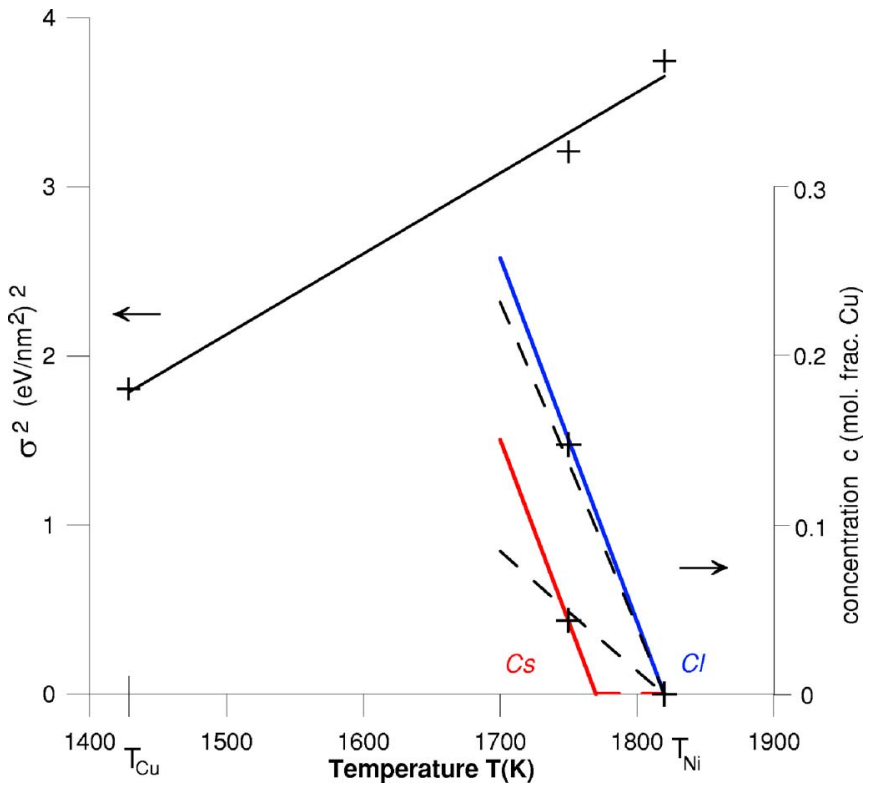

FIG. 2. (Color online) The liquidus $\left(C_{l}\right)$ and solidus $\left(C_{s}\right)$ lines and the square of the interfacial energy $\left(\sigma^{2}\right)$ versus temperature. Crosses represent the experimental (real or numerical) results; the lines are the results of fitting into the linear approximation. The phase boundary $C_{s}=0$ was added because the linear-solution free energy is not singular at $c=0$. Dashed lines - solidus and liquidus of the ideal-solution model.

energy density, $\hat{f}$, along the equilibrium trajectory as a function of the order parameter [Eq. (26)]. The representative point crosses the barrier practically in the middle of the trajectory; this makes the $\mathrm{Cu}-\mathrm{Ni}$ alloy system very similar to a one-component substance. However, contrary to a onecomponent system, the free energy density does not peak at

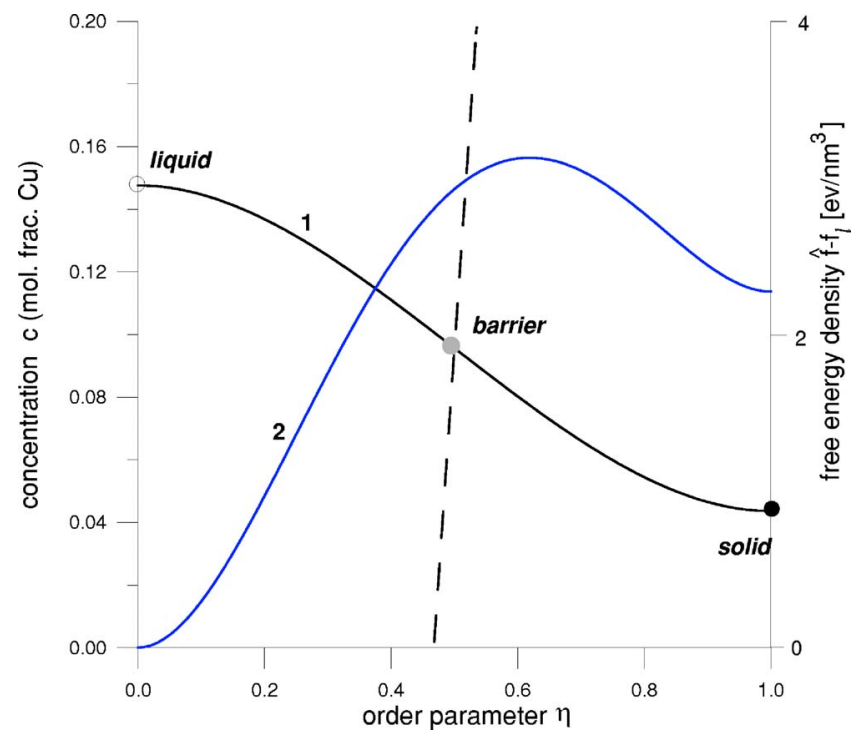

FIG. 3. (Color online) Phase space of the solid/liquid interfacial region in $\mathrm{Cu}-\mathrm{Ni}$ system. Solid line 1,-equilibrium trajectory of the representative point of the system; solid line 2, the free energy density along the equilibrium trajectory as a function of the order parameter; dashed line, position of the free energy barrier. the point where the trajectory crosses the barrier.

From Table I one can see that the chemical contribution, $6 r$, to the barrier-height parameter, $a$, in the solid/liquid $\mathrm{Cu}-\mathrm{Ni}$ system is only $3.6 \%$. The gradient free energy coefficient for this system was found to be $\kappa=3.22 \times 10^{-10} \mathrm{~J} / \mathrm{m}$, which is approximately an order of magnitude greater than the compositional gradient free energy coefficient $\kappa_{\mathrm{cc}} \approx 5.0$ $\times 10^{-11} \mathrm{~J} / \mathrm{m}$ [rescaled for the concentration gradients in Eq. (5)], estimated for coherent interfaces in fcc-based $\mathrm{Ag}-\mathrm{Al}$ alloys. ${ }^{24}$ This may also justify the approximation of $\kappa_{\mathrm{ij}}=0$ used in the present article.

Equation (11) reveals the temperature dependence of the gradient energy coefficient $\kappa$; this may shed light on the thermodynamic nature of the interactions that lead to the formation of the interface. For the purposes of comparing with the theory, the interfacial energies of elemental $\mathrm{Ni}$ and $\mathrm{Cu}$ were included. The interatomic potentials used for the simulations ${ }^{19}$ predict a melting point for pure $\mathrm{Ni}$ of $1820 \mathrm{~K}$ instead of the experimental value of $1728 \mathrm{~K}$ and the interfacial energy $\sigma_{\mathrm{Ni}}=0.310 \mathrm{~J} / \mathrm{m}^{2}$, which is also higher than the value observed experimentally, $0.255 \mathrm{~J} / \mathrm{m}^{2}{ }^{21}$ The melting point and interfacial energy of elemental $\mathrm{Cu}$ were interpolated from the experimental results ${ }^{21}$ because the simulations in Ref. 19 were not performed for elemental $\mathrm{Cu}$; the interpolated values were $T_{\mathrm{Cu}}=1428 \mathrm{~K}$ and $\sigma_{\mathrm{Cu}}=0.215 \mathrm{~J} / \mathrm{m}^{2}$. The square of the interfacial energy was plotted in Fig. 2 as a function of temperature; it fitted into a linear function of temperature with the standard deviation less than 5\%. The fitting into a linear function of temperature allowed us to separate the internal energy and entropy contributions into the gradient free energy: $\kappa=\kappa_{e}-T \kappa_{s}$. Both coefficients turned out to be negative, $\kappa_{e}=-4.88 \times 10^{-10} \mathrm{~J} / \mathrm{m}$ and $\kappa_{\mathrm{s}}=-4.64$ $\times 10^{-13} \mathrm{~J} /(\mathrm{m} \mathrm{K})$ (see Table I). This means that the internal energy effect is to create more interfaces while the entropy effect is to limit the interfacial area and the entropic effect prevails. This conclusion is in qualitative agreement with that of Spaepen ${ }^{22}$ that the main contribution into the solid/ liquid interface tension comes from the entropy decrease due to ordering of liquid near the interface. The latter effect has been recently observed experimentally by $\mathrm{Oh}$ et $a .^{23}$

We realize that the linear chemical approximation does not apply to all alloy systems. Another model, an ideal solution, is often invoked to describe thermodynamic properties of alloys. In Fig. 2, together with the linear-solution model, is shown the phase diagrams of $\mathrm{Cu}-\mathrm{Ni}$ system in the idealsolution approximation (dashed lines): the linear-solution phase diagram envelopes that of the ideal-solution one with the strongest discrepancy near the one-component boundary. In order to verify applicability of our main result, Eq. (19), to an ideal solution, we numerically calculated the surface energies of ideal alloys with properties similar to the $\mathrm{Cu}-\mathrm{Ni}$ system, at different temperatures inside the two-phase region for different magnitudes of the parameter $a / R T_{A}$, where $R$ is the gas constant and $A$ is the low-melting-point component. Figure 4 depicts the quantity $q=\left(36 \sigma_{i d}^{2} / \kappa\right.$ $-a) / 3[C]^{2} \sqrt{\left(f_{l, 2}^{i d} f_{\mathrm{s}, 2}^{i d}\right)}$, which is equal to unity for the linear system. The numerical data show that $q \rightarrow 1.2$ for $a / R T_{A}$ $\rightarrow 0$ when the temperature is not approaching the melting points of pure components of the alloy. The discrepancies 


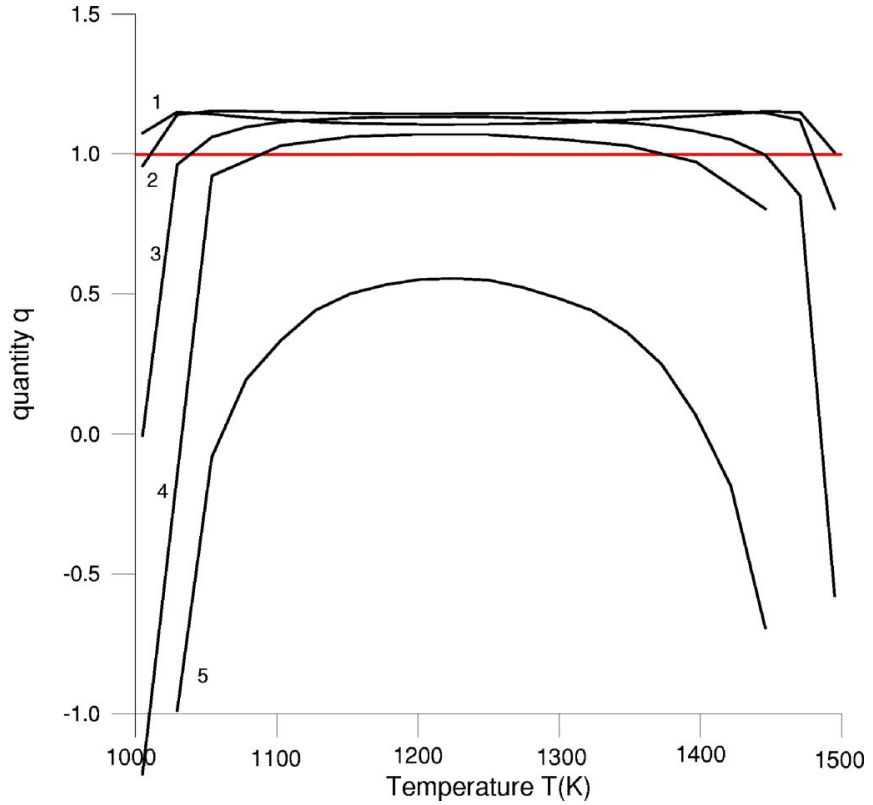

FIG. 4. (Color online) Comparison of the ideal solution and linear chemical models. The quantity $q$ (for the definition see the text) versus temperature for different magnitudes of $a / R T_{A}: 1,0.05$; $2,0.1 ; 3,0.5 ; 4,1.0$; and 5, 5.0. For the linear system $q=1$ (red line).

near the melting points can be easily explained by very different behavior of the models around these temperatures [see Eq. (23) and the discussion below]. Discrepancies of the models at large ratios of $a / R T_{A}$ may be explained by the fact that in this case the representative point of the system "spends more time" in the chemical regions of the phase space, that is close to $\eta=0$ or 1 , and thus is more dependent on the chemical properties of the free energy.

\section{ORDER PARAMETER RELAXATION COEFFICIENT}

As it was explained in the Introduction, finding dynamical parameters of the continuum method presents a more difficult problem than that for the equilibrium ones. Here we intend to identify the relaxation coefficient of order parameter evolution [see Eq. (2)] by relating it to the experimentally measurable solute kinetic coefficient. Therefore we consider stationary motion of a flat interface in an isothermal binary mixture. ${ }^{12}$ Such a situation is described by evolution equations, Eqs. (2) and (3), which in the stationary situation are reduced to

$$
\begin{gathered}
\kappa \frac{d^{2} \eta}{d u^{2}}+\frac{V}{\gamma} \frac{d \eta}{d u}-\frac{\partial f}{\partial \eta}=0 \\
M(\eta) \frac{d}{d u}\left(\frac{\partial f}{\partial c}\right)+V c=\text { const }
\end{gathered}
$$

where the diffusion equation, Eq. (3), has been integrated once. We seek the simultaneous solution of the system of equations, Eqs. (27) and (28), in the form

$$
\begin{gathered}
\frac{d \eta}{d u}=-\frac{4}{\delta} \omega(\eta) \\
c=c_{l}+\left(c_{s}-c_{l}\right) \nu(\eta),
\end{gathered}
$$

where $\delta$ is an undetermined thickness of the moving interface, and $c_{l}, c_{\mathrm{s}}$ are the far-field concentrations in the liquid and solid phases, respectively. Such solution cannot be expected as a long-time dynamical regime in a system with fixed amounts of components, because it does not obey the mass conservation constraint. However, one can expect such solution to apply to initial stages of transformations, like in Ref. 14.

Substitution of the solution, Eqs. (29), into Eq. (27) yields the following solvability conditions:

$$
\begin{gathered}
\delta^{2}=\frac{16 \kappa}{a+3 f_{2}[C]\left(c_{s}-c_{l}\right)} ; \\
V=\frac{3}{4} \gamma \delta f_{2}[C]\left\{\left(c_{l}-C_{l}\right)+\left(c_{s}-C_{s}\right)\right\} .
\end{gathered}
$$

To obtain these conditions we used the same approximation, $H(\eta) \approx 3$, as in the derivation of Eq. (18). Equation (28) can be satisfied simultaneously with Eq. (27) if the solute mobility coefficient is taken in the following form:

$$
M=m \frac{1+2 \eta}{\eta^{2}}
$$

This means that the diffusion of solute in the liquid phase is infinitely fast while that in the solid phase is finite, with the diffusion coefficient in the solid being equal to $D_{s}=3 m f_{2}$. This assumption is reasonable for the solid/liquid $\mathrm{Cu}-\mathrm{Ni}$ system, where $D_{l} / D_{s}=10^{5} .7$ Then the constant in Eq. (28) can be identified as $V c_{\mathrm{s}}$ and we obtain the third solvability condition,

$$
c_{s}-c_{l}=\frac{[C]}{1+\frac{V \delta}{24 D_{s}}} .
$$

Equation (33) establishes the relation between the concentrations in the solid and liquid phases around a moving interface, that is, the kinetic partition coefficient. This condition shows that there is a possibility of solute trapping if the diffusion length in the solid, $D_{s} / V$, is comparable to the thickness of the moving interface $\delta$. Solute trapping, however, is not a primary concern in this paper and will not be dwelled on any further.

Substitution of Eq. (33) into Eq. (30) yields the expression for the thickness of the moving interface,

$$
\delta=\Delta\left(1+\frac{3 V \Delta r}{16 D_{s} a}\right) .
$$

Equation (34) shows that the thickness of a moving interface is only insignificantly different from that of the equilibrium one, $\Delta$. In the derivation of this equation an approximation was used $a \gg r$, which is true for the $\mathrm{Cu}-\mathrm{Ni}$ system (see Table I). 
Substitution of Eq. (33) into Eq. (31) yields the expression for the interfacial velocity, which, to the first order of $\left(V \Delta / D_{s}\right)$, takes the following form:

$$
V=\frac{3}{2} \gamma \Delta f_{2}[C]\left(c_{l}-C_{l}\right) .
$$

Equation (35) identifies the deviation of the concentration in the liquid in front of the moving interface from its equilibrium value, $\left(c_{l}-\mathrm{C}_{l}\right)$, as the "driving force" for interface motion $\left[V=k_{C}\left(c_{l}-C_{l}\right)\right.$, cf. Appendix II in Ref. 14] and establishes the relation between the solute kinetic coefficient $k_{C}$ and the relaxation coefficient $\gamma$,

$$
\gamma=\frac{2 k_{C}}{3 \Delta f_{2}[C]},
$$

This relation is the second main result of the present publication. Direct experimental measurements of the solute kinetic coefficients in binary alloys are still rare due to many technical difficulties. One possible experimental technique was realized in Ref. 14 for $\mathrm{Cu}-\mathrm{Sn}$ systems. The major difference between the $\mathrm{Cu}-\mathrm{Sn}$ and $\mathrm{Cu}-\mathrm{Ni}$ systems is the precipitation and growth of an intermetallic phase in the former one. If we disregard this complication, because the intermetallic layer is very thin, and use the kinetic coefficient obtained in that work, $k_{C}=1 \mathrm{~mm} / \mathrm{s} \mathrm{m}$.f., as an estimate for the $\mathrm{Cu}-\mathrm{Ni}$ system, then the relaxation coefficient comes down to $\gamma$ $=1.37 \times 10^{5} \mathrm{~nm}^{3} / \mathrm{eV} \mathrm{s}\left(8.6 \times 10^{-4} \mathrm{~m}^{3} / \mathrm{J} \mathrm{s}\right)$. The numerical value of the relaxation coefficient allows us to estimate the time scale of the relaxation process in the $\mathrm{Cu}-\mathrm{Ni}$ system: $\tau$ $=1 /(\gamma a) \approx 10^{-7} \mathrm{~s}$. This time scale may be compared with the time scale of the diffusional process: $D_{l} / V^{2}$. The comparison yields the crossover speed, $V_{C}=\sqrt{\left(D_{l} / \tau\right)} \approx 10 \mathrm{~cm} / \mathrm{s}$, which separates the processes controlled by diffusion $\left(V<V_{C}\right)$ from the processes controlled by the interface kinetics $\left(V>V_{C}\right)$.

In addition to presenting an expression for the relaxation coefficient through the solute kinetic coefficient, Eq. (36) establishes a connection between the thermodynamics of a one-component and multicomponent systems. Comparison of Eq. (36) with Eq. (7) shows that the chemical modulus $r$ of alloys is analogous to the latent heat of pure materials.

\section{CONCLUSIONS}

In conclusion, we considered an exactly solvable case of the linear binary and multicomponent systems undergoing a phase transformation and derived equations that relate parameters of the continuum method, like barrier-height $a$, gradient-energy $\kappa$, and relaxation coefficient $\gamma$, to the measurable quantities, like interfacial energy, interfacial thickness, and kinetic coefficient. We found that the contribution of the chemical interactions in the system can be expressed as the renormalization of the barrier-height parameter of the continuum method and replacement of the latent heat with the chemical modulus. Atomic-scale simulations data for a solid/liquid transition in a binary $\mathrm{Cu}-\mathrm{Ni}$ system were chosen for comparison with the theory. The fitting results allowed us to estimate the continuum-method parameters: $a$ $=54.7 \mathrm{eV} / \mathrm{nm}^{3}, \quad \kappa=2.015 \mathrm{eV} / \mathrm{nm}, \quad \gamma=1.37 \times 10^{5} \mathrm{~nm}^{3} / \mathrm{eV} \mathrm{s}$, which can be used for multiscale structure-formation modeling. Comparison of the transition barrier height with the chemical modulus of the $\mathrm{Cu}-\mathrm{Ni}$ system shows that this alloy behaves mostly like a one-component system. Analysis of the temperature dependence of the interfacial energy allowed us to shed light on the magnitudes of the internal energy and entropy contributions into the solid/liquid interface.

\section{ACKNOWLEDGMENTS}

The author is grateful to Mark Asta for providing the simulations data, to Mathis Plapp for a very inspiring discussion, and to Computational Materials Science Network for constructive criticism of the work. This work was supported by a National Science Foundation Grant No. DMR-0244398 from the Material Theory program and ARO Grant No. 46499-MS-ISP from the Materials Science Division.

\section{APPENDIX}

Application of Eq. (4c) to the free energy, Eqs. (1) and (12), yields the following relation:

$$
\begin{aligned}
\frac{\kappa}{2}\left(\frac{d \eta}{d x}\right)^{2} & =\Delta f\left(T ; \rho_{i}, \eta\right)+\frac{1}{2} \rho_{i} N_{i j} \rho_{j}-\frac{1}{2} \rho_{i \alpha} N_{i j} \rho_{j \alpha}-\mu_{i}\left(\rho_{i}-\rho_{i \alpha}\right) \\
& =\Delta f\left(T ; \rho_{i}, \eta\right)+\frac{1}{2}\left(\rho_{i}-\rho_{i \alpha}\right) N_{i j}\left(\rho_{j}-\rho_{j \alpha}\right) .
\end{aligned}
$$

To obtain the last expression in Eq. (A1) we used Eqs. (15) from the text. Substitution of Eq. (16) into Eq. (A1) yields

$$
\frac{\kappa}{2}\left(\frac{d \eta}{d x}\right)^{2}=\Delta f\left(T ; \rho_{i}, \eta\right)+r \nu^{2}
$$

With the help of Eq. (16), the Landau function, Eqs. (13) and (14), can be written as follows:

$$
\Delta f\left(T ; \rho_{i}, \eta\right)=\frac{a}{2} \omega^{2}+\left\{b_{0}+b_{i} \rho_{i \beta}-b_{i}\left[\rho_{i}\right](1-\nu)\right\} \nu .
$$

Substitution of Eq. (15b) into Eq. (A3) yields

$$
\Delta f\left(T ; \rho_{i}, \eta\right)=\frac{a}{2} \omega^{2}+r \nu(1-2 \nu) .
$$

Substitution of Eq. (A4) into Eq. (A2) yields Eq. (17) in the text.
${ }^{1}$ L. D. Landau, Phys. Z. Sowjetunion 11, 26 (1937); see also Collected Papers of L. D. Landau, edited by D. Ter-Haar (Gordon and Breach, London, 1967), p. 202.

${ }^{2}$ L. D. Landau, Phys. Z. Sowjetunion 12, 123 (1937); see also Collected Papers of L. D. Landau, edited by D. Ter-Haar (Gor- don and Breach, London, 1967), p. 236; L. D. Landay and E. M. Lifshitz, Statistical Physics, 3rd ed. (Pergamon Press, Oxford, 1980), p. 471; V. L. Ginzburg and L. D. Landau, Zh. Eksp. Teor. Fiz. 20, 1064 (1950); J. W. Cahn and J. E. Hilliard, J. Chem. Phys. 28, 258 (1958). 
${ }^{3}$ A. Umantsev, Phys. Rev. B 64, 075419 (2001); Phys. Rev. E 69, 016111 (2004).

${ }^{4}$ J. W. Cahn and J. E. Hilliard, J. Chem. Phys. 31, 688 (1959); J. D. Gunton and M. Droz, Introduction to the Theory of Metastable and Unstable States (Springer-Verlag, Berlin, 1983), pp. 61, 63.

${ }^{5}$ H. Metiu, K. Kitahara, and J. Ross, J. Chem. Phys. 65, 393 (1976); S.-K. Chan, ibid. 67, 5755 (1977); A. R. Umantsev and A. L. Roytburd, Sov. Phys. Solid State 30, 651 (1988).

${ }^{6}$ A. A. Wheeler, W. J. Boettinger, and G. B. McFadden, Phys. Rev. A 45, 7424 (1992).

${ }^{7}$ N. A. Ahmad, A. A. Wheeler, W. J. Boettinger, and G. B. McFadden, Phys. Rev. E 58, 3436 (1998).

${ }^{8}$ S. G. Kim, W. T. Kim, and T. Suzuki, Phys. Rev. E 60, 7186 (1999).

${ }^{9}$ L. Gránásy, T. Börzsönyi, and T. Pusztai, Phys. Rev. Lett. 88, 206105 (2002).

${ }^{10}$ B. Echebarria, R. Folch, A. Karma, and M. Plapp, Phys. Rev. E 70, 061604 (2004).

${ }^{11} \mathrm{P}$. Gordon, Principles of Phase Diagrams in Materials Systems (R. Krieger, Malabar, FL, 1983), p. 78; C. H. P. Lupis, Chemical Thermodynamics of Materials (North Holland, New York, 1983), p. 200.

${ }^{12}$ Very often this situation is complicated by morphological instability of the plane front, but this is a completely different matter which will not be addressed in the present article.

${ }^{13}$ A. Umantsev, J. Chem. Phys. 96(1), 605 (1992).

${ }^{14}$ R. A. Lord and A. Umantsev, J. Appl. Phys. 98, 063525 (2005).

${ }^{15}$ J.-C. Toledano and P. Toledano, The Landau Theory of Phase
Transformations (World Scientific, Singapore, 1987).

${ }^{16}$ The Landau function expressed by Eqs. (13) is insignificantly different from the one used in Ref. 3. It was introduced by the author in Ref. 13 for the study of pure systems; later on and independently it was introduced in R. Koboyashi, Physica D 63, 410 (1993), and used for the simulations of phase transformations in binary alloys in Ref. 6.

${ }^{17}$ The quantity $r$ is also related to the quantity $\Gamma$, which was introduced in A. Umantsev and G. B. Olson, Scr. Metall. Mater. 29, 1135 (1993), in order to characterize the rate of coarsening in a multicomponent system.

${ }^{18}$ F. W. J. Olver, Introduction to Asymptotics and Special Functions (Academic Press, New York, 1974).

${ }^{19}$ M. Asta, J. J. Hoyt, and A. Karma, Phys. Rev. B 66, 100101(R) (2002); H. Ramalingam, M. Asta, and A. Van de Walle, Interface Sci. 10, 149 (2002).

${ }^{20}$ Additional data on the free energy potentials were provided by $\mathrm{M}$. Asta.

${ }^{21}$ CRC Handbook of Chemistry and Physics, 86th ed., edited by D. R. Lide (CRC Press, Boca Raton, FL, 2005).

${ }^{22}$ F. Spaepen, Acta Metall. 23, 729 (1975); Scr. Metall. 10, 257 (1976); in Solid State Physics, edited by H. Ehenreich and D. Turnbull (Academic Press, Boston, MA, 1994), Vol. 47, p. 1.

${ }^{23}$ S. H. Oh, Y. Kauffmann, C. Scheu, W. D. Kaplan, and M. Ruhle, Science 310, 661 (2005).

${ }^{24}$ M. Asta and J. J. Hoyt, Acta Mater. 48, 1089 (2000).

${ }^{25}$ D. W. Lewis, Matrix Theory (World Scientific, Singapore, 1991), p. 116. 